\title{
ON THE RADIAL PROJECTION IN NORMED SPACES
}

BY D. G. DeFIGUEIREDO AND L. A. KARLOVITZ ${ }^{1}$

Communicated by F. E. Browder, December 8, 1966

1. Let $X$ be a real normed space with norm \|\|$, T$ the radial projection mapping defined by

$$
T x=x, \quad \text { if }\|x\| \leqq 1, \quad \text { and } \quad T x=x /\|x\|, \quad \text { if }\|x\| \geqq 1 .
$$

Our concern is with the Lipschitz constant of $T$; i.e. with the constant $K$ such that $\|T x-T y\| \leqq K\|x-y\|$ for all $x, y$ in $X$. In particular, we wish to determine under what conditions on the space $X$ the mapping $T$ will be nonexpansive, i.e. $K=1$.

$T$ is a special case of a proximity mapping defined by a convex set in a normed vector space, i.e. a mapping which assigns to each point of $X$, the nearest point of the convex set $C$. There has been a good deal of interest in recent years in proximity maps, nonexpansive mappings, and their interrelations (Moreau, Browder, Petryshyn, Kirk, De Prima, Lions and Stampacchia, and others). It is easy to see that if $X$ is an inner product space (and in particular, a Hilbert space), then $T$ and every proximity map is nonexpansive. More precisely, Kirk and Smiley [1] proved that $X$ is an inner product space if and only if for all nonzero $x$ and $y$ in $X$

$$
\|x /\| x\|-y /\| y\|\| \leqq 2 /(\|x\|+\|y\|)\|x-y\| .
$$

For an arbitrary normed space $X$, Dunkl and Williams [2] have proved that for all nonzero $x, y$ in $X$

$$
\|x /\| x\|-y /\| y\|\| \leqq 4 /(\|x\|+\|y\|)\|x-y\| .
$$

From this it can be seen that $K \leqq 2$. This bound is the best possible because it is easily seen that for $l_{1}, K=2$.

It is of great interest in nonlinear functional analysis to know if there is a normed space which is not an inner product space and for which $K=1$. We prove the following theorem which shows that such spaces exist only for the trivial case of dimension two.

THEOREM. If $X$ has dimension not less than three, then $X$ is an inner product space if and only if $T$ is nonexpansive.

If $X$ has dimension two then the nonexpansiveness of $T$ does not imply that $X$ is an inner product space.

1 The first author was supported by N.S.F. Grant GP-4921, and the second by N.S.F. Grant GP-3666. 
It is also reasonable to ask about the relation of $K$ to other geometric entities of the space. A discussion of this question will appear in another paper.

The theorem is split into the following three propositions which are proved in the sequel.

Proposition 1. $T$ is nonexpansive if and only if $X$ has the following property

(P) For every ordered pair of nonzero vectors $(x, y)$ such that $\|x\|$ $=\|y\|$ and $\left\|x-\lambda_{0} y\right\| \leqq\|x-\lambda y\|$ for all $\lambda$, it follows that $\left|\lambda_{0}\right| \leqq 1$.

REMARK. If $X$ has a strictly convex norm and if it has property (P), then $\left|\lambda_{0}\right|<1$ if $x$ and $y$ are linearly independent. The proof of this fact is similar to the second part of the proof of Proposition 1.

Proposition 2. If $X$ has dimension not less than three, then $X$ has property $(\mathrm{P})$ if and only if $X$ is an inner product space.

Proposition 3. $X$ has property (P) if and only if $X$ has the following property

(J) For every ordered pair of nonzero vectors $(x, y)$ such that $\|x\|$ $\leqq\|x-\lambda y\|$ for all $\lambda$, it follows that $\|y\| \leqq\|y-\lambda x\|$ for all $\lambda$.

Remark. Property $(\mathrm{J})$ expresses that orthogonality in the sense of James [3] is symmetric. Birkhoff [4] discusses property $(\mathrm{J})$ and gives an example of a 2-dimensional space which has this property but which is not an inner product space.

2. Corresponding to each ordered pair of nonzero vectors $(x, y)$ we define the real-valued function

$$
\phi(\lambda)=\phi(\lambda ; x, y)=\|x-\lambda y\|, \quad-\infty<\lambda<\infty .
$$

It follows that $\phi$ is a convex function, it assumes its minimum value for $\lambda$ in a finite interval $\left[\lambda_{1}, \lambda_{2}\right]$, and it is strictly decreasing (increasing) for $\lambda<\lambda_{1}\left(\lambda>\lambda_{2}\right)$. If $X$ has a strictly convex norm, then $\phi$ is a strictly convex function and $\lambda_{1}=\lambda_{2}$. In terms of the function $\phi$, we may restate property $(\mathrm{P})$ as follows.

(P) For every ordered pair of nonzero vectors $(x, y)$, such that $\|x\|=\|y\|$, the function $\phi$ is strictly decreasing (increasing) for $\lambda \leqq-1(\lambda \geqq 1)$.

Proof Of Proposition 1. We first assume that $X$ has property (P) and prove that $T$ is nonexpansive. It suffices to prove the following inequalities

$$
\begin{aligned}
& \|x-y /\| y\|\| \leqq\|x-y\|, \quad \text { for }\|x\| \leqq 1<\|y\| . \\
& \|x /\| x\|-y /\| y\|\| \leqq\|x-y\|, \quad \text { for } 1 \leqq\|x\| \leqq\|y\| \text {. }
\end{aligned}
$$


The inequality (1) is a direct consequence of property $(\mathrm{P})$ applied to the pair $(x,\|x\| /\|y\| y)$. If $1 \leqq\|x\| \leqq\|y\|$, an application of property (P) to the pair $(x /\|x\|, y /\|y\|)$ yields

$$
\|x /\| x\|-y /\| y\|\| \leqq\|x /\| x\|-(\|y\| /\|x\|) y /\| y\|\|=(1 /\|x\|)\|x-y\|,
$$

which implies the inequality (2).

Next we assume that $T$ is nonexpansive and prove that $X$ has property $(\mathrm{P})$. It is sufficient to consider an ordered pair of vectors $(x, y)$ such that $\|x\|=\|y\|=1$ because $|a| \phi(\lambda ; x, y)=\phi(\lambda ; a x, a y)$. For such a pair, the nonexpansiveness of $T$ implies

$$
\|x-y\| \leqq\|x-\lambda y\|
$$

and

$$
\|x+y\| \leqq\|x+\lambda y\|
$$

for all $\lambda \geqq 1$.

Since $\phi(\lambda ; x, y)$ is convex, this shows that $\phi$ is increasing (decreasing) for $\lambda \geqq 1(\lambda \leqq-1)$. To finish the proof we need to show that $\phi$ is strictly increasing (strictly decreasing) for $\lambda \geqq 1(\lambda \leqq-1)$. Suppose, on the contrary, that $\phi$ is not strictly increasing for $\lambda \geqq 1$. Then there exists a constant $d>1$ so that $\phi(\lambda ; x, y)=\phi(1, x, y)$ for $1 \leqq \lambda \leqq d$. Let $z=\alpha x+(1-\alpha) y, 0<\alpha<1$. Then

$$
\begin{aligned}
\phi(1 ; x, z /\|z\|) & =\|x-z /\| z\|\| \\
& =(1-\alpha /\|z\|)\|x-(1-\alpha) /(\|z\|-\alpha) y\| \\
& =(1-\alpha /\|z\|) \phi((1-\alpha) /(\|z\|-\alpha) ; x, y),
\end{aligned}
$$

and

$$
\begin{aligned}
\phi(1+\delta ; x, z /\|z\|)= & \|x-(1+\delta) z /\| z\|\| \\
= & (1-\alpha(1+\delta) /\|z\|) \\
& \cdot\|x-(1+\delta)(1-\alpha) /(\|z\|-(1+\delta) \alpha) y\| \\
= & (1-\alpha(1+\delta) /\|z\|) \\
& \cdot \phi((1+\delta)(1-\alpha) /(\|z\|-(1+\delta) \alpha) ; x, y) .
\end{aligned}
$$

The limit, as $\alpha \rightarrow 0$, of $(1-\alpha) /(\|z\|-\alpha)=1$. Hence we may choose $\alpha>0$ and $\delta>0$ so that

$$
1 \leqq(1-\alpha) /(\|z\|-\alpha) \leqq d,
$$

and

$$
1 \leqq(1+\delta)(1-\alpha) /(\|z\|-(1+\delta) \alpha) \leqq d .
$$

Then it follows, from (3), (4) and the assumption that $\phi(\lambda ; x, y)$ is constant on the interval $[1, d]$, that 


$$
\phi(1 ; x, z /\|z\|)=(1-\alpha /\|z\|) \phi(1 ; x, y),
$$

and

$$
\phi(1+\delta ; x, z /\|z\|)=(1-\alpha(1+\delta) /\|z\|) \phi(1 ; x, y) .
$$

Since $\alpha, \delta>0,(5)$ implies that $\phi(1 ; x, z /\|z\|)>\phi(1+\delta ; x, z /\|z\|)$. This contradicts property $(\mathrm{P})$ for the pair $(x, z /\|z\|)$, and hence $\phi(\lambda ; x, y)$ is strictly increasing for $\lambda \geqq 1$. Similarly we can show that $\phi(\lambda ; x, y)$ is strictly decreasing for $\lambda \leqq-1$, and the proof is finished.

3. Proof of Proposition 2. We may assume that $X$ has dimension three. If $X$ is an inner product space, then it is clear that $X$ has property $(\mathrm{P})$. The proof of the converse is based on a characterization of inner product spaces given by Kakutani [5]. Accordingly it is sufficient to show that for every two dimensional linear subspace $Y$ of $X$ there exists a linear projection $P$ of $X$ onto $Y$ which has norm one. To define such a projection, we choose $x_{0} \in X, x_{0} \in Y$. There exists $y_{0} \in Y$ so that $\left\|x_{0}-y_{0}\right\| \leqq\left\|x_{0}-y\right\|$ for all $y \in Y$. Let $x_{1}=x_{0}-y_{0}$. Every $x \in X$ may be uniquely decomposed in the form $x=\alpha x_{1}+y, y \in Y$. A linear projection $P$, of $X$ onto $Y$, is then defined by $P x=P\left(\alpha x_{1}+y\right)$ $=y$. It remains to show that $P$ has norm one. We consider an arbitrary $x \in X$ with decomposition $x=\alpha x_{1}+y$. If $y=0,\|P x\|=0 \leqq\|x\|$, hence we assume $y \neq 0$. From the construction of $x_{1}$ and the decomposition of $x$, there follows: $\|x-y\|=\left\|\alpha x_{1}\right\| \leqq\left\|\alpha x_{1}-\lambda y\right\|$ for all $\lambda$. Letting $z=(\|x\| /\|y\|) y$, it follows from this inequality that: $\|x-(\|y\| /\|x\|) z\| \leqq\|x-\lambda z\|$ for all $\lambda$. Since $\|x\|=\|z\|$ and $X$ has property $(\mathrm{P})$, this implies $\|y\| /\|x\| \leqq 1$. Hence $\|P x\| \leqq\|x\|$, and the proof is finished.

4. Proof of Proposition 3. We first assume that $X$ has property (P) and prove that $X$ has property (J). Suppose, on the contrary, that there exist nonzero vectors $x$ and $y$ such that $\|x\| \leqq\|x-\lambda y\|$ for all $\lambda$ and such that $\left\|y+\lambda_{1} x\right\|<\|y\|$ for some $\lambda_{1} \neq 0$. Let $m=\left\|y+\lambda_{1} x\right\| /\|y\|<1$. The vectors $y+\lambda_{1} x$ and $m y$ have equal norms. Hence property (P) implies that $\phi\left(1 ; y+\lambda_{1} x, m y\right)<\phi\left(1 / m ; y+\lambda_{1} x, m y\right)$. This inequality implies that $\left\|x-\left((m-1) / \lambda_{1}\right) y\right\|<\|x\|$, which is a contradiction.

To show that if $X$ has property (J) then $X$ has property $(\mathrm{P})$, we consider an arbitrary pair of nonzero vectors $(x, y)$ such that $\|x\|$ $=\|y\|$. Let $\left[\lambda_{1}, \lambda_{2}\right]$ be the finite interval on which $\phi(\lambda ; x, y)$ achieves its minimum. We wish to show that $-1 \leqq \lambda_{1} \leqq \lambda_{2} \leqq 1$. If $\lambda_{1}=\lambda_{2}=0$, we are finished. If not, let $\lambda_{0} \neq 0$ so that $\lambda_{1} \leqq \lambda_{0} \leqq \lambda_{2}$. Let $x_{1}=x-\lambda_{0} y$. Then $\left\|x_{1}\right\| \leqq\left\|x_{1}-\lambda y\right\|$ for all $\lambda$; and it follows from property $(\mathrm{J})$ that 
$\|y\| \leqq\left\|y-\lambda x_{1}\right\|$ for all $\lambda$. If in particular we let $\lambda=-1 / \lambda_{0}$, we may conclude that:

$$
\|y\| \leqq\left\|y-\lambda x_{1}\right\|=\left\|y+\left(1 / \lambda_{0}\right)\left(x-\lambda_{0} y\right)\right\|=\left|1 / \lambda_{0}\right|\|x\| .
$$

Hence $\left|\lambda_{0}\right| \leqq 1$, and the proof is finished.

We wish to thank Professor W. V. Petryshyn for calling our attention to this problem.

\section{REFERENCES}

1. W. A. Kirk and M. F. Smiley, Another characterization of inner product, Amer. Math. Monthly 71 (1964), 890-891.

2. C. F. Dunkl and K. S. Williams, A simple norm inequality, Amer. Math. Monthly 71 (1964), 53-54.

3. R. C. James, Orthogonality and linear functionals in normed linear spaces, Trans. Amer. Math. Soc. 61 (1947), 265-292.

4. G. Birkhoff, Orthogonality in linear metric spaces, Duke J. Math. 1 (1935), 169172.

5. S. Kakutani, Some characterizations of Euclidean space, Japan. J. Math. 16 (1939), 93-97.

UNIVERSITY OF MARYLAND 